\section{$\underset{\substack{\text { hommes } \\ \text { \& migrations }}}{ }$}

Hommes \& migrations

Revue française de référence sur les dynamiques

migratoires

$1293 \mid 2011$

L'immigration dans les musées

\title{
Intégrer le musée dans les approches sur
}

l'immigration

Vers de nouvelles perspectives de recherche

Ramon Grosfoguel, Yvon Le Bot et Alexandra Poli

\section{(2) OpenEdition \\ 12 Journals}

\section{Édition électronique}

URL : http://journals.openedition.org/hommesmigrations/491

DOI : 10.4000/hommesmigrations.491

ISSN : 2262-3353

Éditeur

Musée national de l'histoire de l'immigration

\section{Édition imprimée}

Date de publication : 1 septembre 2011

Pagination : 6-11

ISSN : 1142-852X

\section{Référence électronique}

Ramon Grosfoguel, Yvon Le Bot et Alexandra Poli, «Intégrer le musée dans les approches sur l'immigration », Hommes \& migrations [En ligne], 1293 | 2011, mis en ligne le 31 décembre 2013, consulté le 03 mai 2019. URL : http://journals.openedition.org/hommesmigrations/491 ; DOI :

10.4000/hommesmigrations.491 


\section{Intégrer le musée dans les approches sur l'immigration Vers de nouvelles perspectives de recherche}

Par Ramon Grosfoguel, professeur au Department of Ethnic Studies de UC-Berkeley, Yvon Le Bot, Marie Curie Fellow, Warwick University / European Community (FP7) et Alexandra Poli, chargée de recherche CNRS, CADIS (EHESS)

Durant les dernières décennies, dans divers pays, les pouvoirs publics et les migrants ont ceuvré à la création de musées et d'expositions ayant trait aux questions d'identité, de représentation, de mémoire et d'histoire en relation avec le phénomène migratoire. Parce qu'il est reconnu apte à sauver de l'oubli des objets et des savoirs, et qu'il offre une scène publique, le musée est un lieu très convoité par les mémoires en quête de reconnaissance. Qu'il s'agisse des États-Unis, de l'Amérique latine ou de l'Europe, des créations muséales, plus ou moins récentes, favorisent et renouvellent l'expression d'un droit de mémoire pour différents groupes. Le périmètre de la culture nationale se trouve non seulement élargi mais encore transformé par la présence plus ou moins pérenne de migrants.

Les 25 et 26 juin 2010, nous avons souhaité organiser dans le cadre d'un programme de recherche comparative sur les migrants transnationaux, financé et hébergé par la Fondation Maison des sciences de l'homme $(\mathrm{FMSH})^{(1)}$, deux journées d'études consacrées au thème "Musées et Migrations", bordé par la question de l'autorité culturelle et de l'élaboration des mythes qui ont façonné la perception de la place des immigrés au sein de la culture dominante de différents pays, ainsi que la place de l'interprétation de leur histoire par les immigrés eux-mêmes.

Plus concrètement, le but de cette rencontre était d'amorcer des échanges et de comparer les approches scientifiques sur deux sujets qui sont l'objet de recherches intensives mais dont l'articulation est beaucoup plus inédite. D'un côté, diverses approches se sont en effet développées depuis plusieurs années autour du musée comme terrain d'études. Les museum studies ont ainsi ouvert, comme l'illustre bien la contribution de Marie-Pierre Bera Delaporte à ce dossier, de nouvelles perspectives sur la façon de concevoir le musée comme lieu vivant, traversé par de multiples tensions, 
animé par des publics, tributaire de modes de fonctionnement et de gestion qui le colorent d'aspirations différentes. De l'autre côté, les recherches sur les migrations se sont considérablement diversifiées ces dernières années en s'appliquant à déconstruire l'image figée que nous avons des migrants, qui plus est des immigrés, terme tenace qui emprisonne trop souvent les expériences de ceux que l'on désigne par cette expression. C'est face à ce manque à gagner que plusieurs chercheurs de différentes disciplines se sont mobilisés durant ces deux journées d'échanges afin d'éclairer le destin de plusieurs institutions qui traitent des migrations. Le voyage auquel ils nous invitent fait ressortir de nombreux enjeux propres à l'institutionnalisation de divers pans du passé autour du thème de la migration, le plus souvent recouvert par un flot de débats crispés et de controverses qui se conjuguent à tous les temps en reflétant également un paysage d'approches académiques sur le traitement de la migration dans plusieurs sociétés aux quatre coins de monde. Comment l'histoire de l'immigration s'intègre-t-elle, de façon permanente ou éphémère, au patrimoine muséal d'un pays ? De quelles façons les projets d'institutionnalisation de l'histoire de l'immigration interrogent-ils et transforment-ils le cadre de la nation? Quel est le périmètre historique défini pour présenter l'immigration? Dans certains pays, une partie des migrants sont d'anciens colonisés ou des descendants d'esclaves. Jusqu'à quel point la diffusion du passé de l'immigration est-elle mise en perspective avec l'histoire de l'esclavage et de la colonisation? Autant de questions auxquelles les auteurs de la revue Hommes \& Migrations, pour la plupart issus du champ des études sur les migrations, apportent des précisions et des clefs de compréhension qui se déploient autour de quatre grands axes.

\section{Migrations et "nationalisme muséographique"}

Les musées et expositions consacrés aux immigrations et aux immigrants sont, dans la majorité des cas, prisonniers d'un "nationalisme muséographique". Élaborés à l'aune de la construction du récit national, inscrits dans la perspective de l'intégration nationale, ils en célèbrent la réussite et évoquent parfois ses difficultés ou ses échecs. Il en est ainsi du plus fameux d'entre eux, l'Ellis Island Immigration Museum de New York. C'est aussi le cas du Angel Island Immigration Station Foundation de San Francisco, du Musée de la civilisation de Québec, ou encore du musée Pier 21 d'Halifax. L'Immigration Museum de Melbourne qu'Ilham Boumankhar nous fait découvrir dans ce dossier, et le Migration Museum d'Adélaïde illustrent l'édification de la nation australienne sur la base des vagues d'immigration. Comme en écho à Ellis Island et au Pier 21, un étage entier du musée de Melbourne est consacré au Station Pier du port de Victoria, par lequel transitaient les arrivants. Le Memorial do 
Imigrante de Sao Paulo au Brésil et le Museo de la Inmigración de Buenos Aires en Argentine étudié par Tamar Blickstein sont construits sur le même principe. Les pays mentionnés se considèrent tous comme des "pays d'immigration". Il y semble donc naturel et légitime de célébrer cet acte fondateur. En contraste, la traite des esclaves, immigration forcée ayant également contribué massivement à la formation de certaines de ces nations, est plus rarement prise en compte.

En Europe, on observe en revanche une forte résistance à illustrer le thème de l'immigration. Celui-ci a été longtemps tenu en marge des musées et n'y fait une apparition laborieuse que depuis une ou deux décennies, même s'il faut rappeler comme le fait Benjamin Vanderlick pour la France, le rôle majeur d'initiatives portées par une constellation d'acteurs locaux. Comme dans les "pays neufs", les musées et les expositions qui s'ouvrent au phénomène l'inscrivent dans le processus historique de la formation de l'identité nationale et ne sortent pas du cadre de l'État-nation. Ouverte en 2007, la Cité nationale de l'histoire de l'immigration de Paris l'illustre de manière emblématique par son appellation même, ainsi qu'à travers les débats que sa création a suscités, les expositions et les autres activités qu'elle héberge. Établissement national et financé par l'État, la CNHI n'a d'équivalent dans aucun autre pays européen, même s'il existe quelques initiatives plus ou moins élaborées. Ainsi, l'Immigrant Museum on Danish Immigration (DIM) est un établissement privé, mais financé par la municipalité de Fureso dans la banlieue de Copenhague où il est situé, et se consacre à l'intégration nationale des migrants de diverses provenances, européennes et extraeuropéennes. Le Museo de Historia de la Inmigración de Cataluña ("Museu d'Història de la Immigració de Catalunya" en catalan), créé en 2004 à Sant Adrián del Besós, près de Barcelone, s'intéresse surtout aux migrations en provenance d'autres régions d'Espagne, même s'il a également vocation à inclure la contribution à la formation de l'identité catalane des migrations en provenance de pays étrangers.

Mais l'institutionnalisation culturelle de l'histoire des migrations peut également prendre d'autres formes, et notamment être le fruit d'initiatives communautaires, comme c'est le cas en Belgique, en Grande-Bretagne ou encore aux Pays-Bas, où le Mollocan Museum est dirigé et administré par des immigrés moluquois.

\section{Migrations, musées et expositions communautaires}

Comme le souligne Andrea Meza dans un des articles qui suivent, les migrations d'origine extra-européenne n'occupent qu'une place marginale dans les musées et les expositions en Allemagne. Les quelques événements qui ont eu lieu dans ce domaine lors des dernières décennies constituent des exceptions dans un espace public dominé 
par les questions de la réunification. Parmi les exceptions à caractère institutionnel, la plus importante, le "Projekt Migration"(2005-2006), a donné lieu à plus de cent manifestations, à Cologne et dans diverses villes à travers le pays mais aussi dans quelques pays de départ des immigrés. Ce projet mobilisait des ceuvres d'art contemporaines relatives au fait migratoire, sans toutefois donner véritablement la parole aux migrants eux-mêmes.

Plusieurs établissements et manifestations sont nés en revanche à l'initiative d'immigrés ou de fils d'immigrés turcs, coréens, nigérians... Le Centre de documentation et musée de l'Immigration en Allemagne, Dokumentationszentrum und Museum über die Migration in Deutschland (DOMiT), association privée située à Cologne, a été créé en 1990 par des intellectuels d'origine turque, avec l'objectif de montrer l'apport de l'immigration en provenance de leur pays à la société allemande. Depuis, les collections (photographies, films, enregistrements de musiques et de chansons...) se sont étendues à des migrations d'autres provenances. À Berlin, une association de Coréens, Korientation, a organisé, à l'occasion du vingtième anniversaire de la réunification de l'Allemagne, une exposition, Shared. Divided. United, mettant en parallèle la situation des deux pays. Les musées, expositions et centres culturels conçus et gérés par des membres de communautés issues de l'immigration ou du transfert forcé de populations sont sans doute plus nombreux aux États-Unis qu'en Europe. Le Mexican Fine Arts Center Museum de Chicago, devenu le National Museum of Mexican Art (NMMA), qui figure parmi les institutions étudiées par Cristina Castellano, a été fondé dans les années quatre-vingt au sein de la communauté des Mexicains-Américains très nombreux dans cette ville. Il se propose de donner à voir une "mexicanité" plongeant ses racines dans le passé préhispanique, se déroulant à travers l'histoire du Mexique, celle des Chicanos (citoyens américains d'origine mexicaine) et des migrants des dernières décennies. Il s'agit de présenter des figures de l'imaginaire d'un "nationalisme culturel" se réclamant d'une autre origine que celle, européenne, de la société majoritaire. Le changement de dénomination qui fait de cet établissement un musée national américain, recevant des subventions étatiques et se voyant assigner une mission qui déborde le simple cadre communautaire, est-il un épisode de l'affrontement de deux nationalismes ou peut-il être considéré comme un pas vers la reconnaissance de la culture mexicaine comme composante du multiculturalisme américain, au même titre que d'autres cultures longtemps tenues pour minoritaires voire mineures, comme celle des Noirs ou des Indiens?

\section{Migrations, esclavage, colonialisme}

En Europe, quelques établissements d'apparition récente consacrent la totalité ou une partie de leurs activités à produire et à diffuser des connaissances sur l'histoire du "commerce triangulaire", de l'esclavage et de son abolition, tels que l'International 
Slavery Museum de Liverpool et l'Institute for the Study of Dutch Slavery Past and Legacy (NINSEE) d'Amsterdam. Ces espaces amènent à s'interroger, comme nous y invite Stephen Small à travers l'exemple de Liverpool, sur la visibilité et l'invisibilité des héritages de l'esclavage et de la colonisation que portent les migrations dans des musées d'anciennes puissances coloniales mais également sur le rôle des groupes concernés par cette histoire dans leur création et leur développement.

Dans son article, Véronique Bragard montre qu'en Belgique les expositions relatives à l'immigration restent lestées d'un passé colonial qui ne "passe" pas, soit-il glorifié ou occulté. Elle signale toutefois quelques tentatives récentes pour réévaluer ce passé et traiter de la question de la diaspora congolaise.

En France, le choix d'établir la CNHI dans le Palais de la Porte Dorée a contribué à associer histoire de l'immigration et colonisation, à mettre ce lien au premier plan, alors que toutes les immigrations de l'époque de l'industrialisation ne proviennent pas des pays colonisés ou anciennement colonisés, et qu'une grande partie de celles présentées à la Cité proviennent de pays européens. Pourtant, l'objectif de certains des concepteurs et organisateurs a sans doute été d'opérer un renversement.

\section{Individus globalisés, sujets migrants}

Le traitement de l'immigration dans les musées et les expositions est orienté par des logiques nationales, communautaires et/ou post-coloniales (ces dernières incluant, dans certains cas, le thème de l'esclavage).Les analyses comprises dans ce dossier sont également guidées par ces logiques. En filigrane, cependant, on entraperçoit la possibilité d'autres approches plus appropriées, plus à même de rendre compte de l'expérience des migrants, y compris celle des migrants transnationaux de la période actuelle. Ceux-ci sont absents des musées et des analyses proposées qui, sauf rares exceptions, se placent dans une perspective historique, regardent le passé.

Certains responsables du Musée de l'Immigration de Cologne, inspirés par Ulrich Beck, sont conscients de la nécessité de dépasser le cadre de l'État-nation et de se situer dans la perspective de "sociétés transnationales, en Europe et dans le monde, au-delà des appartenances nationales et ethniques. [...] Dans une société d'immigration, les identités résultent de l'hétérogénéité, du croisement des frontières et de l'échange interculturel "2". Mais il ne s'agit pas seulement d'élargir le spectre, de se situer sur une scène globale. Rendre compte des expériences des migrants demande aussi de se libérer des visions qui enferment les migrants dans des catégories identitaires. La projection du paradigme colonial sur le phénomène migratoire est importante mais a aussi ses limites. Comme le souligne Françoise Vergès dans un article portant sur un centre culturel-musée à l'île de la Réunion, "la recherche ne peut se contenter d'être une mise en accusation des 
faiblesses de l'adversaire ou l'établissement d'une liste de bêtises, déclarations ou actes

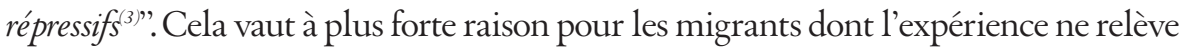
pas des rapports coloniaux, ne s'inscrit pas dans le contexte de la mondialisation opérée par les empires coloniaux, mais dans celui de la globalisation néo-libérale.

Une façon d'échapper à cette réduction est de s'intéresser à la subjectivité des migrants, à la diversité de leurs expériences, au caractère multiple et mouvant de leurs identités, à leur capacité à choisir entre elles et à en inventer de nouvelles par croisements et créations, à leurs luttes contre les discriminations, les rapports de domination et d'exclusion, à leurs initiatives et productions culturelles.

Les créations artistiques et culturelles des migrants transnationaux, surtout lorsqu'ils sont circulants, n'ont pas fait leur entrée, sinon exceptionnellement et comme par effraction, dans les musées, qui restent animés, sinon par la glorification des empires coloniaux du passé, du moins par un souci national ou communautaire, par l'objectif d' inscrire l'immigration dans le récit national (4)" ou d'illustrer et de perpétuer une identité et une mémoire communautaires.

Les musées tendent à exacerber la tension entre la mémoire comme souvenir et la mémoire comme instrumentalisation du souvenir. Aussi est-ce en dehors des musées, dans des espaces plus ouverts, moins institutionnalisés, ou à l'occasion d'expositions temporaires, que s'exposent le plus souvent les productions culturelles des migrants. Aujourd'hui comme par le passé, les migrations permettent à des idées, à des représentations du monde, des connaissances et des comportements de circuler et de se développer dans de nombreuses sociétés. L'étude des musées de l'immigration soulève les questions de la mise en récit du changement des peuples, du croisement des mémoires, de l'exploration progressive d'une identité collective, de la place de chaque groupe d'immigrés au sein de la société nationale. Là encore, la comparaison entre différents contextes sera l'occasion de faire sortir du champ institutionnel des musées l'étude des transformations du paysage muséal à la lumière des enjeux liés aux migrations, à la diversité, au multiculturalisme.

\section{Notes}

1. Nous remercions les responsables de la FMSH pour leur soutien scientifique et financier.

2. Museum International, 2008 , p. 137.

3. Françoise Vergès, "Le musée postcolonial : un musée sans objets", in Nicolas Bancel, Florence Bergnault, Pascal Blanchard, Ahmed Boubeker, Achille Mbembe, Françoise Vergès, Ruptures postcoloniales. Les nouveaux visages de la société française, Paris, La Découverte, 2010, p. 474.

4. Michel Wieviorka, "Inscrire l'immigration dans le récit national", in Alexandra Poli, Michel Wieviorka, Jonna Louvrier, "La Cité nationale de l'histoire de l'immigration. Quels publics?", Hommes e Migrations, hors-série, octobre 2007. 